Revue

Revue de l'histoire des religions

de Ihistoire des religions

\title{
À propos de la construction d'un débat sur les mythes égyptiens
}

Reflections on the construction of the discussions on Egyptian myths

\section{Youri Volokhine}

\section{OpenEdition \\ Journals}

Édition électronique

URL : http://journals.openedition.org/rhr/9328

DOI : $10.4000 /$ rhr.9328

ISSN : 2105-2573

\section{Éditeur}

Armand Colin

\section{Édition imprimée}

Date de publication : 1 décembre 2018

Pagination : 619-644

ISBN : 978-2-200-93200-8

ISSN : 0035-1423

Référence électronique

Youri Volokhine, «À propos de la construction d'un débat sur les mythes égyptiens », Revue de l'histoire des religions [En ligne], 4 | 2018, mis en ligne le 01 décembre 2020, consulté le 13 janvier 2021. URL http://journals.openedition.org/rhr/9328 ; DOI : https://doi.org/10.4000/rhr.9328 


\section{À propos de la construction d'un débat sur les mythes égyptiens*}

Le mythe est une catégorie problématique dans le champ de l'égyptologie. Depuis au moins le XIX siècle, les égyptologues ont utilisé cette catégorie, apparemment intraduisible en égyptien, pour rendre compte de différentes formes de savoir, sacerdotales ou littéraires par exemple. Il s'agit ici non pas de proposer une nouvelle approche théorique sur l'objet "mythe", mais plutôt de tenter un parcours dans l'histoire des interprétations de cette notion. On discutera donc des propositions et des idées formulées par les égyptologues, pour présenter de manière synthétique un débat, qui conduit non seulement vers les théories modernes en histoire des religions, mais qui renvoie encore à l'histoire même de la notion de mythe dans le savoir occidental et éventuellement aux contacts antiques entre savoirs grecs et égyptiens.

\section{Reflections on the construction of the discussions on Egyptian myths}

Myth is a problematic category in Egyptology. At least since the 19th century, Egyptologists have used this category, which is apparently untranslatable in ancient Egyptian, to describe various forms of knowledge, priestly or literary for example. It is not the purpose here to offer a new theoretical approach to myth, but rather to try to shed light on the history of its interpretation. We shall thus discuss the ideas formulated by Egyptologists in order to present a synthetic view of the question. By so doing, we will address not only the issue of modern theories used by specialists in the field of religious studies, but also that of the history of the notion of myth in western knowledge and possibly of the relationship and contacts between ancient Greek and Egyptian hermeneutics.

* Le présent texte est une version remaniée d'une contribution parue antérieurement: "À propos de la construction d'un débat sur les mythes égyptiens », Le savoir des religions. Fragments d'historiographie religieuse, éd. Daniel Barbu, Philippe Borgeaud, Mélanie Lozat, et al., Gollion, Infolio, 2014, p. 471-498. Je remercie les éditeurs qui m'ont autorisé à publier ici une nouvelle version de ce texte. 
Aborder la question des mythes, voire d'une mythologie, en contexte polythéiste, est un exercice plus redoutable qu'il n'en a l'air de prime abord. Les hellénistes nous l'ont appris, à l'instar de Marcel Detienne : poser le mythe comme objet d'étude, une vieille affaire dans la pensée occidentale, demande quelques précautions. Très récemment, Philippe Matthey a montré toute l'actualité de la question à propos de plusieurs récentes publications ayant pour objet la mythologie grecque ${ }^{1}$. L'objet du présent article est de se pencher sur un autre débat concernant le statut du mythe, celui qui s'énonce depuis le XIX ${ }^{\mathrm{e}}$ siècle dans le cadre de l'égyptologie.

\section{LE MYTHE COMME OBJET DE SCANDALE OU DE DÉDAIN}

L'étude des mythes ne fut pas exempte de préjugés de la part des égyptologues, qui, jadis, semblaient parfois s'en méfier. Or, dans une certaine mesure, cette méfiance procédait d'un jugement en partie négatif sur le polythéisme égyptien dans son ensemble, que l'on tendait à considérer comme une masse confuse. On ne reviendra pas ici sur les travaux des exégètes de l'Europe moderne, qui avaient déjà centré une partie de leur réflexion sur une Égypte que l'on ne lisait encore qu'au travers des sources classiques ou bibliques; ni sur les efforts des philosophes des Lumières, ou des premiers égyptologues à la suite de Champollion; mais commençons cavalièrement au début $\mathrm{du} \mathrm{xx}^{\mathrm{e}}$ siècle, moment où l'égyptologie a déjà bien forgé ses armes philologiques et constitué une bonne partie de son corpus : on y verra clairement se poser les données du problème. En 1905, Adolf Erman (1854-1937)², publie une imposante Religion der Ägypter, dans laquelle il exprime, dès la première page, un sentiment particulier sur l'objet de son étude :

«Une chose rend malaisée notre juste appréciation de la religion égyptienne : elle entraîne derrière elle, du moins dans sa forme officielle, toutes les sottises de ses débuts; on ne peut vraiment demander à

1. Philippe Matthey, «Étudier les mythes en contexte francophone. À propos de quatre ouvrages récents », Kernos, t. 29, 2016, p. 1-15.

2. Sur ce savant, voir Bernd U. Schipper (éd.), Ägyptologie als Wissenschaft. Adolf Erman (1854-1937) in seiner Zeit, Berlin-New York, De Gruyter, 2006. 
personne de s'enthousiasmer pour une telle barbarie. Celle-ci surgit pour nous au premier plan, mais en réalité, pour les Égyptiens d'une époque plus évoluée, elle ne constituait qu'un arrière-plan dont l'importance ne dépassait pas, dans leur véritable vie religieuse, l'importance que d'autres attachent aux dogmes apportés par la tradition ${ }^{3}$. »

Sans doute, en ce début du $\mathrm{xx}^{\mathrm{e}}$ siècle, le monde du mythe et de la multitude des dieux - où l'étrangeté de la zoomorphie saute aux yeux - était-il encore pour ce grand philologue celui de l'aberration. Adolf Erman ne pouvait probablement pas admettre que ce monde apparemment confus soit pourtant aussi celui des sages prêtres égyptiens. Sous cet aspect, le brillant philologue n'était sans doute pas encore dégagé d'une très vieille fascination occidentale pour une secrète Égypte des mystères, celle des temples secrets, des prêtres reclus et des initiations, en contraste avec une Égypte insensée, celle de la zoomorphie divine et de la frénésie populaire, par exemple $\mathrm{e}^{4}$ Une autre illustration que je souhaiterais donner de cette sorte de mouvement de recul face au foisonnement du polythéisme égyptien, concerne un autre ancien maître de la philologie égyptienne. Gustave Lefebvre (1879-1957), publiant en 1946 ses Romans et contes, considérait que :

«(les Égyptiens) ayant créé des dieux à leur propre image, (...) ont admis tout naturellement que ceux-ci menaient la même vie médiocre, pratiquaient les mêmes vertus moyennes, avaient les mêmes mœurs que l'humanité ; ils leur ont même supposé des vices que nous considérons généralement comme honteux ${ }^{5}$. »

Alors que, pour ce philologue (helléniste de formation), les Grecs auraient toujours conservé quelque respect par rapport à leurs dieux dans leur mythologie, les Égyptiens se seraient montrés irrespectueux et sarcastiques. On lirait même dans cette mythologie le récit d'actions indécentes «dignes de Rabelais », « ou de Boccace »; ainsi, poursuit Gaston Lefebvre :

«On peut se demander quelle estime les Égyptiens, à toutes époques, avaient pour des dieux si vulgaires, en quoi consistait la piété

3. Adolf Erman, La religion des Égyptiens, Paris, Payot, 1952 (traduction française de Henri Wild d'après la troisième édition allemande de 1934), p. 17.

4. Erik Hornung, L'Égypte ésotérique. Le savoir occulte des Égyptiens et son influence en Occident, Paris, Alphée, 2007 (traduction française de Das esoterische Aegypten, Munich, Beck, 1999).

5. Gustave Lefebvre, Romans et contes égyptiens de l'époque pharaonique, Paris, Maisonneuve, 1946, p. 179. 
des masses dont un tel conte faisait les délices, et si la religion ne se ramenait pas en définitive, pour la plupart des Égyptiens, à la croyance en l'efficacité de la magie ${ }^{6}$. »

La perspective qu'il défendait distingue la pieuse et intellectuelle religion de l'élite de la vulgaire et populaire magie, forme inférieure de la religion, bonne pour les masses. Cette querelle sur la valeur « religieuse » de la magie n'était pas neuve. Dans les systèmes d'interprétation $\mathrm{du} \mathrm{XIX}^{\mathrm{e}}$, la magie tendait à apparaître comme une phase antérieure à la vraie religion. C'était l'opinion de James Frazer. Pour Émile Durkheim, la démarcation est différente : la magie était exclue de cette «église » qui représentait pour lui la religion communautaire. Or, tout se passe comme si l'objet «mythe » souffrait de façon analogue de préjugés du même acabit ${ }^{7}$. Dans le champ de l'égyptologie, cette manière de rejeter implicitement le mythe dans la «fable», ou de le subordonner à la magie, imprégnait encore assez profondément les théories des savants de la première moitié $\mathrm{du} \mathrm{XX}^{\mathrm{e}}$ siècle. Ainsi, dans le célèbre manuel écrit par Étienne Drioton (1889-1961) - chanoine formé à la théologie au Vatican avant de devenir un brillant égyptologue - et Jacques Vandier (1904-1973), publié initialement en 1938 et souvent réimprimé depuis, considère-t-on qu'une haute doctrine, assimilée à une gnose monothéiste, était l'apanage de la haute classe en Égypte ; elle coudoyait le curieux assemblage des autres opinions polythéistes, «fétichistes ou symbolistes $»^{8}$. En fait, derrière ce débat entre religion d'élite et religion vulgaire, se dessine en filigrane tout le dossier du prétendu « monothéisme égyptien », qui aurait été la religion des «sages » et de l'élite : une religion que l'on imagine non pas mythologique mais philosophique. Pour Étienne Drioton et Jacques Vandier, la mythologie est au contraire populaire, familière, triviale ; elle a «saveur de folklore »; elle renvoie l'image d'une humanité pleine de faiblesses et de passions ${ }^{9}$. Cette idée se lit en fait dès les prémices de l'égyptologie au XIX ${ }^{\mathrm{e}}$ siècle, toute jeune discipline

6. Ibid., p. 179.

7. Marcel Detienne, L'invention de la mythologie, Paris, Gallimard, 1981.

8. Étienne Drioton et Jacques Vandier, L'Égypte. Les peuples de l'Orient méditerranéen, Paris, PUF ( Clio. Introduction aux études historiques», t. 1), 1938 (4e éd., 1962), p. 63.

9. Ibid., p. 72-73. 
alimentée évidemment par la lecture des auteurs classiques, et aussi par certaines théories du $\mathrm{XVIII}^{\mathrm{e}}$ qui étaient encore bien vivaces. Jean-François Champollion lui-même, lecteur (entre autres) de Jablonski ou du citoyen Dupuis, cherche, comme ses devanciers, l'Être Suprême derrière la multiplicité apparente des dieux ${ }^{10}$. Cette vision sera celle de presque tous les pionniers de l'égyptologie.

En 1893, Gaston Maspero faisait paraitre le premier volume du recueil de ses œuvres qu'il assemblait sous le nom d' «Études de mythologie et d'archéologie égyptiennes ». La «mythologie» est donc bien au programme, à cette époque où fleurissait justement la « mythologie comparée » de Friedrich Max Müller. Cependant, il s'agit de tout autre chose ici que d'une entreprise comparatiste, ou d'un travail qui se vouerait foncièrement à l'étude des mythes :

« Je ne me suis pas adonné par goût aux études religieuses; les circonstances m'ont obligé à m'y livrer. En premier lieu, mes cours à l'École des Hautes Études et au Collège de France, quelque soin que je prisse de les faire porter au principal sur des matières d'histoire et de grammaire, touchaient souvent au dogme et à la mythologie de l'Égypte $(\ldots)^{11}$. »

Pour Maspero, il est évident que la science des religions est une discipline auxiliaire de la philologie, de l'archéologie ou de l'histoire : elle est un outil. Toutefois, il poursuit en montrant combien son appréciation de la religion égyptienne, qui était d'abord orientée, suite à ses années d'étudiant, vers la certitude de l'unité du Dieu égyptien (celui des sages) - «tout était Soleil pour moi comme pour mes maîtres » (à savoir Lepsius, Chabas, Déveria, de Rougé, etc.), s'est totalement modifiée au contact des monuments égyptiens (notamment «les quatre mille lignes » des Textes des Pyramides), qui lui montrèrent que «les Égyptiens eux-mêmes ne semblaient avoir jamais professé, ni seulement soupçonné, la plupart des belles doctrines qu'on leur prêtait si généreusement $\gg^{12}$. À ses propres yeux, il avait contribué à enlever la patine qui lustrait les idées qu'on se faisait sur la religion des Égyptiens, cette prétendue « vieille sagesse ».

10. Jean Yoyotte, «Champollion et le Panthéon égyptien », Bulletin de la Société Française d'Égyptologie, t. 95, 1982, p. 76-108.

11. Gaston Maspero, Études de mythologie et d'archéologie égyptienne, t. I, Paris, Leroux («Bibliothèque Égyptologique », t. 1), 1893, p. V.

12. Ibid., p. VI. 
On ne peut ici, faute d'espace, s'attarder sur la redécouverte d'Akhénaton. Soulignons néanmoins que la découverte de sa théologie, focalisée sur le dieu Aton, fut interprétée très tôt comme le «premier monothéisme» de l'humanité13. Or, dans la pensée des chercheurs de l'aube du $\mathrm{XX}^{\mathrm{e}}$ siècle, cette religion d'un dieu «unique» dessine un paysage duquel le mythe est absent. Adolf Erman, à la suite des premiers exégètes de la «religion d'Akhénaton » (dont Henry Breasted, le traducteur des hymnes de Tell el-Amarna), pensait qu'Akhénaton avait apporté à l'Égypte « une foi nouvelle», «une réforme», « une doctrine », « une croyance monothéiste », sous forme d'une révolution, avant que la réaction anti-amarnienne n'amène le pays à la décadence ${ }^{14}$ : pour Erman, la religion d'Akhénaton débarrasse l'Égypte du «fatras » accumulé par les millénaires; elle dénote une tendance monothéiste qui serait celle de l'élite, incomprise par un peuple enraciné dans le paganisme ${ }^{15}$. Ce regard sur l'Égypte était bien entendu tout teinté de perspective chrétienne, laquelle tend à minimiser l'importance du mythe.

De fait, les égyptologues en activité dans le premier quart $\mathrm{du} \mathrm{xx}^{\mathrm{e}}$ siècle ne tentaient pas vraiment de réfléchir sur la nature du mythe en Égypte, mais souhaitaient reconstituer les fondements idéologiques de la culture pharaonique, en se livrant à une reconstruction de sa « religion primitive », comme le fit par exemple Kurt Sethe ${ }^{16}$. Toujours en Allemagne, d'autres philologues et épigraphistes, comme Hermann Junker, se plongeaient toutefois dans les mythes - désignés indifféremment comme Legende ou Sagen. Hermann Junker, avec ses travaux portant notamment sur les textes ptolémaïques, reconstruit les récits épars du «mythe de la déesse lointaine », en assemblant toutes les bribes qu'il parvient à tirer des monuments ptolémaïques et romains de

13. Pour ce qui concerne la question du monothéisme amarnien, voir ma contribution «Atonisme et monothéisme : quelques étapes d'un débat moderne », Akhénaton et Néfertiti. Soleil et ombres des pharaons, Genève, Musée d'Art et d'Histoire, 2008, p. 129-141.

14. A. Erman, La religion des Égyptiens, p. 160.

15. Ibid., p 157.

16. Kurt Sethe, Urgeschichte und älteste Religion der Ägypter, Leipzig, Deutsche morgenländische Gesellschaft («Abhandlungen für die Kunde des Morgenlandes », t. 18, fasc. 4), 1930. 
Philae et des temples nubiens, notamment ${ }^{17}$; Kurt Sethe, encore, se plonge aussi bien dans les textes démotiques ${ }^{18}$ que dans les textes ptolémaïques au sein desquels il reconstruit le système cosmogonique thébain, et s'illustre par des études fondatrices ${ }^{19}$. Mais l'arrière-plan théorique d'une réflexion sur le mythe n'intéresse pas franchement ces savants philologues, éditeurs de textes et défricheurs de la littérature religieuse tardive. Lesquels philologues furent assez peu sensibles aux théories explicatives des faits religieux en vigueur alors. En revanche, dans le monde francophone des années 1920-1930, les hypothèses «totémistes » ou « fétichistes » (émanant notamment de l'anthropologie anglosaxonne) vont beaucoup influer sur les travaux d'Alexandre Moret $^{20}$ et imprégner aussi les idées du Neuchâtelois Gustave Jéquier ${ }^{21}$. Mais, encore, ce n'est pas tellement le(s) mythe(s) qui intéresse(nt) ces derniers : pour Jéquier, il serait même "vain de chercher dès maintenant à établir un système théologique ou mythologique, tel que le concevaient les égyptologues du siècle dernier» (notamment Maspero) ${ }^{22}$ : il s'agit plutôt de comprendre «les idées », les «croyances », les « doctrines ». Bref, point de «mythes» (en tant qu'objets spécifiques d'étude) selon cette perspective. De même, Jacques Vandier, que nous avons déjà cité, renonce implicitement à utiliser les mots de «mythes», voire de «mythologie», dans sa Religion égyptienne, qu'il publie en $1944^{23}$. Il y traite des « légendes », liées à des « cycles » (solaires,

17. Hermann Junker, Der Auszug der Hathor-Tefnut aus Nubien, Berlin, Verlag der Königlichen Akademie der Wissenschaften (« Abhandlungen der KöniglichPreussichen Akademie der Wissenschaften »), 1911 ; Die Onurislegende, Vienne, Alfred Hölder («Denkschriften der Kaiserlichen Akademie der Wissenschaften. Philosophisch-historische Klasse », t. 59, fasc. 1-2), 1917.

18. Kurt Sethe, Zur altägyptischen Sage vom Sonnenauge, das in der Fremde war, Leipzig, J. C. Hinrichs ( « Untersuchungen zur Geschichte und Altertumskunde Aegyptens », t. 5, fasc. 3), 1912.

19. Kurt Sethe, Amun und die Acht Urgötter von Hermopolis, Berlin, Verlag der Akademie der Wissenschaften («Abhandlungen der Preussischen Akademie der Wissenschaften, Philosophisch-Historische Klasse », t. 4), 1929.

20. Voir spécialement Alexandre Moret, Mystères égyptiens, Paris, Armand Colin, 1923.

21. Voir par exemple Gustave Jéquier, Considérations sur les religions égyptiennes, Neuchâtel, La Baconnière, 1946.

22. Ibid., p. 9.

23. Jacques Vandier, La religion égyptienne, Paris, PUF (« Mana-Introduction à l'histoire des religions », t. 1), 1944. 
osiriens), légendes divines «qui sont au peuple ce que la théologie est aux prêtres $»^{24}$; il y analyse les contes, la théologie, liée à des « systèmes » (héliopolitain, hermopolitain, etc.). De même, dans son édition du papyrus Jumilhac, Vandier préfère généralement user du terme «légende » plutôt que de mythe, employant avec parcimonie l'appellation de "géographie mythologique ${ }^{25}$. Cette formulation n'est pas sans liens avec cette «Kult-Topographie » à laquelle on s'adonnait depuis un moment ; une démarche qui, pour partie, procède de l'indispensable inventaire, mais qui présente le risque aussi de se muer en une sorte de dissection de l'Égypte en une myriade d'entités locales. On pourrait néanmoins penser que cet émiettement dans l'approche de la documentation, qui éloigne la réflexion de la synthèse, témoigne d'une raisonnable prudence nécessaire pour aborder les fondements intellectuels du système. Prudence que l'on pourrait détecter notamment, à la même époque, dans le très beau Dictionnaire de la civilisation égyptienne édité par Georges Posener, aidé de Serge Sauneron et Jean Yoyotte, en 1957, dans lequel ne figure aucune entrée sub voce «mythes » ou «mythologie $»^{26}$.

\section{À LA RECHERCHE D'UNE CATÉGORIE ÉGYPTIENNE D'EXPRESSION DU MYTHE}

Cette sorte de réticence ou de prudence à envisager le mythe comme un sujet en soi sur lequel travailler en Égypte ancienne, dont nous venons de voir quelques exemples, est révélatrice de certains présupposés encore communs chez les égyptologues de la première moitié du $\mathrm{XX}^{\mathrm{e}}$ siècle. L'univers des mythes, comme nous l'avons également rappelé, avait tendance à se voir placé dans le fourre-tout de la magie, bric-à-brac par essence irrationnel. Cette méfiance s'est éteinte peu à peu, au moins depuis les travaux d'Henri Frankfort (1897-1954). Doué d'une grande ouverture d'esprit - qui l'avait mené de la pratique à la théorie, des chantiers archéologiques égyptiens et irakiens à l'Oriental Institute de Chicago (où il fut professeur), puis à l'Institut Warburg à Londres

24. Ibid., p. 36.

25. Jacques Vandier, Le papyrus Jumilhac, Paris, éditions du CNRS, 1960.

26. Terme utilisé cependant passim, et que l'on trouve dans l'index (p. 319). 
(qu'il dirigea de 1949 jusqu'à sa mort en 1954) - Frankfort avait proposé une approche des religions égyptienne et assyrienne pour en retrouver «l'esprit» (il évoquait un mode de pensée «mythopoétique »), en faisant table rase des idées préconçues, et, surtout, en observant les catégories indigènes ${ }^{27}$. Si ce thème de la «mythopoétique » n'est pas sans rappeler quelques anciennes théories sur le mythe, et particulièrement celle de Karl Otfried Müller $^{28}$, il faut reconnaître que l'appel à l'observation des «catégories indigènes » était salutaire. La plupart des synthèses savantes sur la religion égyptienne qui ont suivi procèdent en général de cette approche, comme, plus tard, le livre exemplaire d'Erik Hornung, Der Eine und die Vielen ${ }^{29}$. Peu avant l'ouvrage de Frankfort, Siegfried Schott avait publié (en 1945) une monographie, sous forme de vaste panorama des textes religieux, mettant en avant le «mythe»: Mythe und Mythenbildung ${ }^{30}$. Schott posait fondamentalement que le «mythe » était caché dans les textes égyptiens, textes qui l'utilisent sous forme d'allusions et de jeux de mots. D'emblée, dès les textes les plus anciens (les Textes des Pyramides), les Égyptiens auraient posé les bases de ce système allusif, développant sans cesse au cours de leur histoire religieuse l'art de la citation, préféré à celui de la narration. Quelques années plus tard, dans une importante monographie sur la religion, Siegfried Morenz (1914-1970), qui était théologien de formation, tend à considérer la «pensée mythique » comme un stade de la pensée qui se mit en place au début de la période

27. Henri Frankfort, Kingship and the Gods. A Study of Ancient Near Eastern Religion as the Integration of Society and Nature, Chicago, University of Chicago Press, 1948. Cf. aussi Id., Before Philosophy: the Intellectual Adventure of Ancient Man. An Essay on Speculative Thought in the Ancient Near East, Chicago, University of Chicago Press, 1946.

28. Voir à ce propos M. Detienne, L'invention de la mythologie, p. 228.

29. Erik Hornung, Der Eine und die Vielen, Darmstadt, Wissenschaftliche Buchgesellschaft, 1971 ; Id., Les dieux de l'Égypte. Le Un et le multiple, Paris, Le Rocher, 1986. Cette traduction française, réimprimée par la suite en livre de poche (chez Champs Flammarion), comporte malheureusement des erreurs.

30. Siegfried Schott, Mythe und Mythenbildung im alten Ägypten, Leipzig, J.C. Hinrichs ( « Untersuchungen zur Geschichte und Altertumskunde Aegyptens », t. 15), 1945. Cf. aussi les travaux de Eberhard Otto, dont: Das Verhältnis von Rite und Mythus im Ägyptischen, Heidelberg, C. Winter ( Sitzungsberichte der Heidelberger Akademie der Wissenschaften. Philosophisch-Historische Klasse », t. 42, fasc. 1), 1958. 
historique ${ }^{31}$. Pour lui, le mythe se couple au rite, qui l'actualise. Morenz prend en compte cet aspect non narratif des sources qui révèlent les mythes en Égypte : selon lui, «aucun mythe n'a été mis pour lui-même sous forme littéraire $»^{32}$; au contraire, le mythe, le plus souvent, serait « mis au service d'une fin étrangère (...), la magie». Le mythe n'aurait pas été conçu en Égypte autrement qu'en fonction de sa dépendance (couplage) avec le culte ; ce «stade de la pensée » fournirait ainsi le matériel dans lequel on vient piocher, comme dans une boîte à outils - ces idées, on le verra encore ci-dessous, sont largement partagées par les égyptologues.

Mais, si le mythe est une "boîte à outils », encore faudrait-il en décrire l'ordonnance interne. Ce qui n'est pas simple, et l'on y a souvent renoncé. Un peu réfractaires à un comparatisme trop universaliste, assez peu enclins à accepter des modèles interprétatifs formulés en anthropologie ou en histoire des religions et mis au point dans d'autres champs culturels, certains égyptologues se sont parfois défendus pour mieux mettre en avant la spécificité de leur terrain nilotique. Ainsi, Emma Brunner-Traut, dans son article «Mythos » du Lexikon der Ägyptologie, affirme d'emblée qu' « un vocable égyptien pour désigner le mythe est inconnu $»^{33}$. Mais l'absence de mot pour désigner la chose n'implique pas son inexistence. Emma Brunner-Traut voulait souligner qu'aucune catégorie de récits égyptiens, ni aucun genre littéraire pratiqué par les Égyptiens, ne recouvre ce que l'on appelle «mythe ». Ce qui nous ramène au point de départ (incontournable) : qu'est-ce qu'un «mythe»? Je doute bien entendu que la réponse soit simple. En revanche, il est manifeste que l'ensemble des textes religieux égyptiens est sous-tendu d'une armature d'historiettes (historiolae) impliquant les dieux. C'est de ce côté que le «mythe » doit sûrement être recherché. Une véritable galaxie de fragments, d'allusions, d'histoires jamais entièrement racontées, mais apparemment bien connues. Ainsi, pour reconstituer les

31. Siegfried Morenz, La religion égyptienne. Essai d'interprétation, Paris, Payot, 1984 (traduction française de Ägyptische Religion, Stuttgart, W. Kohlhammer Verlag [« Die Religionen der Menschheit », t. 8], 1960).

32. S. Morenz, op. cit., p. 280.

33. Emma Brunner-Traut, Lexikon der Ägyptologie, t. IV, Wiesbaden, Harrassowitz, 1982, s.v. « Mythos », col. 277-286. 
mythes égyptiens - les récits sur les dieux -, les chercheurs se sont appliqués à la longue pratique du regard croisé entre les textes et les époques, pour organiser et reconstituer une énorme matière, et pour l'ordonner afin de la rendre présentable au lecteur moderne ${ }^{34}$. L'organisation scientifique de cette mythologie égyptienne tend à dégager des cycles de légendes : par exemple, le cycle osirien et le cycle horien (destin d'Osiris, luttes entre Horus et Seth), le cycle de la «déesse dangereuse » (l'œil de Rê, le massacre de l'humanité, le retour de la «lointaine», etc.) Ces cycles entrent dans différents cadres: récits cosmogoniques, cosmologiques, aventures des « dieux sur terre », entre autres. À un degré différent, tous ces récits orientent vers certaines thématiques clés : domaine régalien (transmission du pouvoir), perpétuité de la vie, entretien du monde et victoire sur les forces néfastes. $\mathrm{Si}$, pour nous, cette approche thématique de la mythologie égyptienne permet de nous y retrouver, il y a tout lieu de croire que ce type d'approche classificatoire est assez éloigné de la réflexion égyptienne. Dans le sillage de la réception de la pensée sur le mythe de Claude LéviStrauss, qui toucha l'ensemble des sciences humaines, il se trouve que fort peu d'égyptologues ont ressenti le besoin de questionner leur matière par le biais d'un modèle d'analyse structuraliste. Certains, cependant, ont compris l'apport stimulant que pouvait avoir une certaine forme de structuralisme appliqué à l'étude des mythes égyptiens. Philippe Derchain, notamment dans les brillants articles qu'il rédigea pour le Dictionnaire des Mythologies d'Yves Bonnefoy, s'est illustré dans cette veine, cherchant la «logique interne de la pensée mythique » des Égyptiens ${ }^{35}$. Pour Derchain, il s'agit de « reconnaître la nature d'un signe mythique égyptien, c'est-à-dire de définir le signifié porté par un signe ou signifiant (...) à l'intérieur d'une construction théologique égyptienne $»^{36}$.

34. L'ouvrage commun de Dimitri Meeks et Christine Favard-Meeks, La vie quotidienne des dieux égyptiens, Paris, Hachette, 1993, constitue l'une des plus belles réussites en la matière.

35. Philippe Derchain, Dictionnaire des mythologies et des religions des sociétés traditionnelles et du monde antique, éd. Yves Bonnefoy, Paris, Flammarion, 1981, p. 325. Voir les articles «Anthropologie»; «Cosmogonie»; «Divinité »; « Mort »; « Rituels égyptiens ».

36. Philippe Derchain, Hathor Quadrifrons. Recherches sur la syntaxe d'un mythe égyptien, Istambul, Nederlands Historisch-Archaeologisch Institut in het Nabije Oosten, 1972, p. 1. Derchain revendique ici son inspiration structuraliste, 
Auparavant, en 1970, Derchain soulignait encore que «l'inventaire systématique des mythes égyptiens n'a pas été fait, ni leur étude entreprise $»^{37}$. Pour Derchain, les mythes doivent être appréhendés comme des «essais poétiques de représenter les phénomènes naturels et sociaux»; la théologie égyptienne s'exprime "par combinaisons de mythes et de symboles ». Il faut donc comprendre la logique combinatoire.

Cet appel de Philippe Derchain pour cibler le mythe et en faire un terrain de recherche prioritaire pour l'égyptologie n'est pas resté lettre morte; ces dernières années, de nombreuses contributions y ont été consacrées. En 1977, Jan Assmann empoigne le problème et propose un tableau original sur la nature du mythe en Égypte. Selon lui, il convient notamment de délimiter trois catégories d'expression du mythe dans le cadre d'un schème généalogique ${ }^{38}$ : (1) le récit de base, le «mythe » («GenoTexte », narratif et oral); (2) puis, sa différentiation fonctionnelle («Derivation», son usage opérationnel, par exemple dans les rituels écrits); (3) enfin, le récit mythologique («mythische Aussage »), qui est la réalisation narrative concrète du mythe écrit («Phäno-Texte »). Jan Assmann, dans ce schéma, oppose l'oralité (celle du «mythe ») à l'écriture, par laquelle nous connaissons le « récit mythique ». Le mythe génère sa réalisation écrite. Assmann laisse entendre, sans le préciser toutefois, que c'est la mémoire collective - l'inconscient collectif -, qui génère le mythe. Pour Assmann, il faudrait donc distinguer le mythe de base de ce que nous connaissons... ou plutôt de ce que nous méconnaissons. En effet, ni les récits narratifs (rares) mettant en scène les dieux (cf. par exemple «Le mythe de la vache du ciel»), ni les «contes mythologiques » (cf. par exemple «Le conte des deux frères »), ni les allusions mythologiques (les «dérivations fonctionnelles» d'Assmann) dont fourmillent les textes religieux, ne nous suffisent pour avoir une idée vraiment représentative du monde du mythe en Égypte, tel qu'il pouvait se présenter pour un Égyptien (instruit) de

citant Claude Lévi-Strauss et Ferdinand de Saussure, ainsi que Jean-Pierre Vernant, Marcel Detienne, et évoquant, dans le champ de l'égyptologie, Herman te Velde.

37. Philippe Derchain, Histoire des Religions, éd. Henri-Charles Puech, t. I, Paris, Gallimard («Encyclopédie de la Pléiade »), 1970, p. 108.

38. Jan Assmann, «Die Verborgenheit des Mythos im Alten Ägypten», Göttinger Miszellen, t. 25, 1977, p. 7-43. 
l'Antiquité. Pour Assmann, il faut penser que cette situation reflète un phénomène historique. Si je le suis bien, il faudrait comprendre que l'Égypte est une culture qui s'enracine dans une haute période lors de laquelle la séparation entre le monde des dieux et des hommes n'était, en quelque sorte, pas achevée (le roi-dieu prédynastique). Le mythe, en tant que discours, nécessiterait cette séparation (dont il rend compte); et c'est ainsi qu'il n'y aurait pas eu au sens propre de mythes avant le Nouvel Empire (la théologie ayant évolué) ; ou plutôt, avant cette période, les mythes n'étaient pas assemblés en récit pouvant être racontés (ou écrits) de façon suivie $^{39}$. Cela dit, il faut bien préciser que cet article d'Assmann sur le mythe est relativement ancien, et que désormais cet auteur poursuit, en profondeur, d'autres pistes d'analyses, notamment dans ses derniers livres consacrés, entre autres, aux rapports entre mythe, histoire et mémoire. Jan Assmann a récemment forgé le concept de $«$ mythomoteur $»^{40}$. Pour lui «le mythe d'Horus et de Seth mue le souvenir d'une dualité originelle en énergie symbolique, en élan vers l'établissement toujours renouvelé d'une unité » : ce serait, donc, le «mythomoteur» qui serait «le secret de la singulière stabilité de l'État égyptien», un renforçateur de «l'identité collective». Il serait trop long ici de discuter les références théoriques qui alimentent la réflexion d'Assmann, notamment les travaux de Jan Vansina, Maurice Halbwachs ou Max Weber, ou, en ce qui concerne l'Égypte ancienne, le lien que l'on pourrait tracer avec l'idée, notamment défendue par Erik Hornung, d'une Geschichte als Fest ${ }^{41}$.

L'idée diffuse que le monde mythologique des Égyptiens est un fouillis, auquel ces derniers n'avaient finalement recours, et avec parcimonie, que pour soutenir d'autres activités (rites, littérature,

39. Voir aussi Jan Assmann, «Die Zeugung des Sohnes: Bild, Spiel, Erzählung und das Problem des ägyptischen Mythos », Funktionen und Leistungen des Mythos. Drei altorientalische Beispiele, éd. Jan Assmann, Walter Burkert et Fritz Stolz, Fribourg-Göttingen, Universitätsverlag et Vandenhoeck \& Ruprecht («Orbis Biblicus et Orientalis », t. 48), 1982, p. 13-61.

40. Jan Assmann, La mémoire culturelle. Écriture, souvenir et imaginaire politique dans les civilisations antiques, Paris, Aubier, 2010 (édition originale en allemand : Munich, C. H. Beck, 1992), p. 154-155.

41. Erik Hornung, Geschichte als Fest : Zwei Vorträge zum Geschichtsbild der frühen Menscheit, Darmstadt, Wissenschaftliche Buchgesellschaft («Libelli», t. 246), 1966 ; texte repris notamment dans L'esprit du temps des Pharaons, Paris, Lebaud, 1996, p. 147-163, avec des compléments bibliographiques, p. 214-215. 
magie), n'a pas forcément disparu de l'égyptologie. Mais, à vrai dire, l'impression de confusion du monde mythologique égyptien provient certainement de l'usage que l'on fait des sources. En effet, quiconque (non égyptologue de formation) fait l'expérience d'ouvrir, par exemple, une traduction en langue moderne du Livre des Morts, a de quoi être déçu, me semble-t-il, s'il s'avisait d'y rechercher les contours d'un mythe bien dessiné et bien narré. On pourrait penser que cette opinion négative repose sur l'idée qu'un mythe, en Égypte ou ailleurs, est essentiellement une narration (orale, d'abord, éventuellement écrite ensuite) ${ }^{42}$, à l'appui de raisons théoriques que l'on peut certes parfaitement comprendre ${ }^{43}$. À cet égard, l'égyptologie ne fait que marcher dans les sillons de la majorité des interprétations du mythe qui, comme le rappelait Nicole Belmont, mènent à une recherche du mythe exclusivement dans le langage $»^{44}$. Une approche qui relie Friedrich Max Müller à Roland Barthes dans une longue chaîne d'herméneutes dépend fondamentalement d'une approche «hellénocentrée » (le mythos est d'abord et essentiellement une parole). Pour John Baines ${ }^{45}$, le mythe en Égypte est «a sacred or culturally central narrative ». La plupart de ces «narratives» concernent les dieux et se déroulent à l'époque où ceux-ci régnaient sur terre. Baines remarque aussi que des mythes héroïques sur la fondation de l'État ne sont pas attestés, alors qu'ils sont rapportés néanmoins par les auteurs classiques (la tradition manéthonienne sur Ménès, par exemple). En Égypte, les mythes sont intégrés dans des compositions religieuses (comme les hymnes) qui n'ont pas de structures narratives. À la différence de la Mésopotamie ou de

42. Cf. par exemple l'opinion emblématique de Gerardus van der Leeuw, La religion dans son essence et ses manifestations, Paris, Payot, 1948 (édition originale en néerlandais : Groningen, 1933), p. 404 : « Le mythe (...) par essence fait l'objet de récits, de répétitions sans cesse énoncées ».

43. Vincent Arieh Tobin, «Myths : An Overview », The Oxford Encyclopedia of Ancient Egypt, éd. Donald B. Redford, t. II, Oxford, Oxford University Press, 2001, col. 464-469, col. 464.

44. Nicole Belmont, Paroles païennes. Mythe et folklore, Paris, Imago, 1986, p. 10.

45. John Baines, "Myth and Literature", Ancient Egyptian Literature. History and Forms, éd. Antonio Loprieno, Leyde-New York-Cologne, Brill («Probleme der Ägyptologie », t. 10), 1996, p. 361-377. Du même auteur, voir notamment : «Egyptian Myth and Discourse ; Myth, Gods, and the Early Written and Iconographic Records », Journal of Near Eastern Studies, t. 50, 1991, p. 81-105. 
la Grèce, peu de récits mythiques narratifs sont préservés. Le seul long récit narratif ancien connu (datant de la XVIII ${ }^{e}$ dynastie) est ledit récit de la «Destruction de l'humanité » (ou le mythe de la Vache du Ciel $)^{46}$. Il existe quelques attestations de cette littérature qualifiée de «contes », non seulement dans les textes du Nouvel Empire (le conte d' "Horus et de Seth», le «conte des Deux Frères », etc.), mais encore très bien représentée dans la littérature démotique (avec le conte de "Setné $»^{47}$, par exemple, ou dans le dit «Mythe de l'œil du Soleil $»^{48}$ ). Les narrations mythologiques sont également rares dans les inscriptions des temples (voir néanmoins le grand «Mythe d'Horus » à Edfou ${ }^{49}$ ) ; enfin, il existe une série de papyrus datant de l'époque ptolémaïque qui recensent des réalités cultuelles et mythologiques locales (comme le papyrus Jumilhac ${ }^{50}$ ou le $«$ papyrus du Delta $\left.»^{51}\right)$. Cette carence relative en récits narratifs mythiques a suscité beaucoup de discussions : certains ont pensé que les Égyptiens n'avaient en somme que peu de mythes. D'autres, dont John Baines, pensent que «nous ne pouvons pas accéder aux mythes eux-mêmes $»^{52}$, mais que nous n'avons accès qu'à leur transformation, leur emploi secondaire. Le mythe égyptien serait donc une «entité hypothétique». Les mythes sont intégrés dans la littérature, et leur contenu y est traité librement. En outre, toujours selon John Baines, il faut aussi souligner qu'en Égypte les formes «non continues » d'écritures ont la primauté. Considérant que la plupart des approches font la part trop belle à une conception du mythe en tant que « récit

46. Erik Hornung, Der ägyptische Mythos von der Himmelskuh. Eine Ätiologie des Unvollkommenen, Fribourg-Göttingen, Universitätsverlag et Vandenhoeck \& Ruprecht (« Orbis Biblicus et Orientalis », t. 46), 1982.

47. Voir dernièrement: Damien Agut-Labordère et Michel Chauveau, Héros, magiciens et sages oubliés de l'Égypte ancienne. Une anthologie de la littérature en égyptien démotique, Paris, Les Belles Lettres, 2011, p. 19-65.

48. Françoise de Cenival, Le mythe de l'Eil du Soleil, Sommerhausen, G. Zauzich (« Demotische Studien », t. 9), 1988.

49. Herbert-Walter Fairman, The Triumph of Horus. An Ancient Egyptian Sacred Drama, Londres, B. T. Batsford, 1974.

50. J. Vandier, Le papyrus Jumilhac, 1960.

51. Dimitri Meeks, Mythes et légendes du Delta d'après le papyrus Brooklyn 47.218.84, Le Caire, Institut français d'archéologie orientale (« Mémoires publiés par les membres de l'Institut français d'archéologie du Caire », t. 125), 2006.

52. J. Baines, « Myth and Literature», p. 374. 
littéraire $»^{53}$, Jürgen Zeidler ${ }^{54}$ a entrepris d'appliquer la méthode de Vladimir Propp ${ }^{55}$, combinée avec une méthode structuraliste, s'attachant à repérer des "mythèmes ». Ces petites unités, sous formes de «mythes minimaux» seraient la forme de base du mythe en Égypte: elles possèdent leur dynamique propre. Les récits structurés et développés, qui puisent dans ce matériel (cf. encore ci-dessous pour cette idée), ne sont qu'un développement littéraire et historique ultérieur. Cela n'empêche pas Christiane Zivie-Coche de souligner le problème de l'usage «très extensif » du terme «mythe », qui n'est pas toujours très «adéquat $»^{56}$, et de préciser : «l'appréciation du divin par les Égyptiens s'est faite moins par la mise en scène narrative de métaphores mythiques que par un procès plus conceptuel et spéculatif, d'où la pauvreté des mythes lorsque l'on considère globalement le tableau religieux de l'Égypte ». Pour Pascal Vernus, le mythe est une narration : « une narration mettant en jeu des êtres dans un temps présenté comme radicalement différent de son contexte d'énonciation et censé véhiculer une part du savoir d'une société sur le monde $»^{57}$. Cela étant, si le mythe est narration, alors il demande à être recherché dans des textes dont la finalité n'est nullement d'en faire l'exposé. Vernus distingue le mythe «dans la religion collective », c'est-àdire le mythe "dans le savoir religieux stocké pour lui-même", du «mythe dans les manifestations de la religion funéraire », et

53. En revanche, il faut relever que l'on a choisi d'appeler «papyrus mythologiques » les papyrus funéraires illustrés de la XXI ${ }^{e}$ dynastie, depuis leur publication par Alexandre Piankoff et Natacha Rambova: Mythological Papyri, New York, Pantheon Books («Bollingen Series », t. 40, fasc. 3), 1957. Pour Rambova, ces documents contiennent, sous forme illustrée, des « symbolic representations of condensed mythological conceptions» (op. cit., p. 3). N'oublions pas que Rambova, égyptologue à ses heures en marge de sa carrière hollywoodienne (elle fut, en outre, l'épouse de Rudolf Valentino), était notoirement sous l'influence des doctrines spiritualistes et ésotériques.

54. Jürgen Zeidler, «Zur Frage der Spätentsehung des Mythos im Ägypten », Göttinger Miszellen, t. 132, 1993, p. 85-109.

55. Comme le relève Pascal Vernus, «La notion de mythe dans la civilisation pharaonique », Cuadernos de Filosofía, t. 9-10, 2001, p. 26-27.

56. Christiane Zivie-Coche, «Qu'est-ce qu'un dieu ? », Hommes et dieux en Égypte, éd. Françoise Dunand et Christiane Zivie-Coche, Paris, Cybèle, 2006 (2 éd.), p. 60-65.

57. P. Vernus, "La notion de mythe dans la civilisation pharaonique ", p. 11-33. Cf., du même auteur, Dictionnaire amoureux de l'Égypte pharaonique, Paris, Plon, 2009, s.v. « Mythe », « Mythes en œuvre» et « Mythes (typologie des récits) », p. 650-672. 
$\mathrm{du}$ « mythe dans les manifestations de la religion individuelle non funéraire ». Dans chacune de ces catégories, il isole un certain nombre de supports qui mettent en œuvre le mythe. Le mythe «pour lui-même» se trouve ainsi narré dans certains «traités monographiques » (ainsi la dite « pierre de Chabaka » contenant un célèbre texte de «théologie memphite ${ }^{58}$ ), dans des traités relatifs aux cultes locaux (comme le papyrus Jumilhac), dans des papyrus faisant la «somme des traditions cultuelles » (comme le «Livre du Fayoum ${ }^{59}$ ). Dans les textes funéraires, le mythe est utilisé, selon Vernus, au «second degré »; il s'agit d'un détournement menant de la «religion collective » (où s'invente en quelque sorte le mythe) au bénéfice du défunt, qui doit, dans l'expérience de la mort, vaincre les périls de l'Au-delà. Plusieurs modalités de détournement ou d'emploi sont d'ailleurs attestées. Enfin, il arrive aussi que l'on puisse utiliser le mythe dans la sphère de la vie quotidienne, dans la médecine par exemple, ou la magie. Vernus, enfin, propose d'utiliser le terme de «Mythe» (ou, mieux encore, en le distinguant clairement par la typographie : MYTHE), dans un sens restreint et spécifique à la civilisation égyptienne, comme une «succession narrative sise à un temps distinct de l'énonciateur », ou dans un «présent intemporel ». Pour être vraiment « Mythe », il faut en outre un enchaînement d'unités minimales (soit de manière lâche, soit hiérarchisée).

\section{MYTHE ET LITTÉRATURE}

Penchons-nous brièvement sur un aspect de la question que nous avons vu affleurer à plusieurs reprises : la mise en écriture des mythes sous forme d'histoire «narrative». On rencontre effectivement des mythes dans cette littérature égyptienne que l'on trouve traduite sous les titres de «Romans et contes», ou

58. En dernier lieu, sur ce texte fameux, voir Benedikt Rothöler, Neue Gedanken zum Denkmal memphitischer Theologie, Heidelberg, Université d'Heidelberg («Maat Monographie », t. 1), 2006 (http://www.ub.uni-heidelberg. de/archiv/7030).

59. Horst Beinlich, Das Buch vom Fayum : Zum religiösen Eigenverständnis einer ägyptischen Landschaft, Wiesbaden, Harrassowitz (« Ägyptologische Abhandlungen », t. 51), 1991; Jean Yoyotte, «Le grand livre du Fayoum», AnEPHE V $\mathrm{V}^{\mathrm{e}}$ section, t. 96,1987 , p. 155-162. 
simplement de «contes»(Tales, Märchen, etc.). Il se trouve que la traduction en langue moderne de l'ensemble de ce corpus hétéroclite n'occupe pas beaucoup plus d'une centaine de pages. Cette rareté ne s'explique pas seulement par l'éventualité de la perte de cette littérature, mais peut-être, comme l'a proposé Pierre Grandet, par le fait que ces textes, qui sont le produit d'une catégorie particulière de la population, celle des scribes, étaient peut-être réservés à un degré inférieur de l'éducation et de l'apprentissage de ces derniers ${ }^{60}$. Dans ce cas, selon Grandet, il faudrait les considérer comme des exercices stylistiques, qui puisent dans des genres différents : démonstration de rhétorique (conte du Paysan plaideur), récits populaires merveilleux (récits du papyrus Westcar), ou roman historique (aventures de Sinouhé au Proche-Orient). Parmi ces textes, il existe des récits qualifiés de $«$ contes mythologiques $»^{61}$. Les exemples les plus connus sont les aventures d'Horus et Seth (sur le papyrus Chester Beatty I), ou encore le célèbre "conte des deux frères ». Mais certains textes de ce genre résistent toutefois à entrer dans une seule catégorie. Pensons au papyrus des «dieux contre la Mer» (ou légende d'Astarté) ${ }^{62}$, qui est un récit mythologique (peut-être d'inspiration proche-orientale), mettant en scène un dieu sauveur de l'Égypte, que le texte met en relation avec le roi Amenhotep II. Mais il s'agit aussi, et surtout (peut-être) d'une sorte de texte de "propagande ", comparant en fin de compte l'action salvatrice d'une divinité toutepuissante avec celle du roi guerrier. Les intentions publicitaires du texte sont évidentes, et il y a d'ailleurs peu de chances que ce type de texte émane d'un exercice élémentaire de scribe. Les « contes mythologiques » partagent une structure commune assez simple: des personnages divins s'affrontent, physiquement ou verbalement. Ces textes narratifs apparaissent au Moyen Empire, mais sont surtout représentés à la XVIII ${ }^{e}$ dynastie, puis à l'époque ramesside. Or, ces récits ont, d'une certaine façon, été longtemps dévalorisés par l'égyptologie, comme nous l'avons vu au début de notre exposé : Alan Gardiner, Gustave Lefebvre, ou encore, par

60. Pierre Grandet, Contes de l'Égypte ancienne, Paris, Hachette, 1998, p. IV-V.

61. J. Baines, « Myth and Literature », p. 365-366.

62. Sur le genre littéraire de ce texte, Philippe Collombert, Laurent Coulon, «Les dieux contre la mer. Le début du "papyrus d'Astarté" (P. BN 202) », Bulletin de l'Institut Français d'Archéologie Orientale, t. 100, 2000, p. 222-226. 
exemple, Edda Bresciani, s'accordaient sur le fait que la trivialité des récits les situait dans une sphère «profane »; on ne voulait pas y reconnaître la littérature égyptienne sous sa plus belle expression. Les aventures qu' on y lit ne seraient que les « reflets d'un monde humain vicieux $\gg^{63}$ : en bref, une littérature populaire, écrite pour le délassement. Ces considérations réductrices sont surannées. Dans son étude du papyrus Chester Beatty I (le conte d'Horus et Seth), Michèle Broze a montré que ce texte célèbre n'était pas l'adaptation littéraire d'un mythe déformé pour créer une œuvre de divertissement, mais un texte témoignant d'une réelle pratique du mythe ${ }^{64}$ : comme dans d'autres récits comparables, la finalité d'Horus et Seth est idéologique, car il s'agit, en mobilisation les récits sur les dieux, d'établir l'autorité du roi. Autrement dit, il est insuffisant de penser que les «contes mythologiques» sont des dérivations à partir d'un récit de base qui nous est inconnu car relevant de l'oralité. En effet, l'écriture rend sans doute complexes les données orales, en les soumettant à des impératifs idéologiques.

\section{MYTHÈMES ET MATÉRIELS MYTHOLOGIQUES}

Dans son étude sur les cosmogonies égyptiennes ${ }^{65}$, Susanne Bickel propose d'abandonner toutes les idées préconçues voulant enfermer a priori le mythe égyptien dans des définitions trop serrées : ainsi, elle s'oppose à l'idée que le terrain du mythe soit d'abord celui de l'oralité6 ${ }^{6}$. Pour Bickel, le mythe égyptien est un objet savant, un instrument complexe de réflexion et est le «produit d'une élite instruite ». S'il est évident que le(s) mythe(s) étaient discutés sans doute dans toutes les classes sociales, Bickel se demande toutefois si «les réponses fournies par l'imaginaire populaire ne correspondaient pas toujours exactement aux explications développées par les doctes » : ceux-ci se servaient des

63. Voir les remarques (critiques) de Michèle Broze, Mythe et roman en Égypte ancienne. Les aventures d'Horus et Seth dans le papyrus Chester Beatty I, Louvain, Peeters («Orientalia Lovaniensia Analecta », t. 76), 1996, p. 2 et suivantes.

64. M. Broze, Mythe et roman en Égypte ancienne, p. 221.

65. Susanne Bickel, La cosmogonie égyptienne avant le Nouvel Empire, Fribourg-Göttingen, Universitätsverlag et Vandenhoeck \& Ruprecht ( Orbis Biblicus et Orientalis », t. 134), 1994, p. 265-283.

66. S. Bickel, La cosmogonie égyptienne avant le Nouvel Empire, p. 278. 
«mythèmes » existants ${ }^{67}$ pour les intégrer dans des compositions funéraires (notamment), et, de ce fait, les développaient. Le mythe, pour Bickel «est l'un des principaux moyens d'expression du discours théologique $»^{68}$, il est un "langage dans lequel la science et avec elle la spéculation peuvent s'exprimer et se développer », le moyen favori d'expression « des philosophes et des théologiens ». Il y a quelques années, Dimitri Meeks, dans son édition du «papyrus du Delta ${ }^{69}$, a entrepris de redéfinir théoriquement la question du mythe; il s'interroge, d'une part, sur la date d'émergence des mythes, c'est-à-dire celle de leur mise en écriture, et se demande si le mythe en Égypte a vraiment eu vocation à être mis en écriture. En effet, il n'y a pas, comme le rappelle Meeks, de corpus mythique narratif qui soit structuré. Il faut donc pour le chercheur moderne parcourir l'ensemble des textes - gigantesque tâche - quelle que soit leur nature, pour repérer les éléments identiques ou similaires, puis les assembler. Ensuite, il s'agit de réaliser que le contenu est plus vaste que les simples énoncés. Considérer que le mythe est une narration (avec prémices, développement, conclusion) est contestable, car cette appréciation repose sur l'idée de textes codifiés et intouchables. La civilisation pharaonique, pour Meeks, a fonctionné sur un modèle radicalement différent. Il faut aussi reconnaître que l'Égypte est une civilisation qui a inventé son écriture, et que l'on peut suivre le processus de découverte. Cette invention de l'écriture se déroule sur plusieurs siècles : c'est au Moyen Empire qu'émergent simultanément des récits sapientiaux et mythiques : ceci n'est pas un hasard. Ce fait révèle, selon Meeks, une maîtrise progressive du langage écrit, autorisant l'émergence de genres nouveaux. Ce que l'Égypte nous offre comme matériel mythique se présente sous formes de «briques narratives »: des phrases clés, représentant l'articulation la plus petite d'un mythe. On les accole; on les habille (pour arriver parfois à une narration ${ }^{70}$ ). Cette façon de faire du mythe se démarque de la notion lévi-straussienne de «mythème »; en effet,

67. Ibid., p. 281.

68. Ibid., p. 283.

69. D. Meeks, Mythes et légendes du Delta d'après le papyrus Brooklyn 47.218.84, p. 163 et suivantes.

70. Katja Goebs, «A Functional Approach to Egyptian Myth and Mythemes », Journal of Near Eastern Religions, t. 2, 2002, p. 27-59. 
les mythèmes de Lévi-Strauss sont plutôt des paquets de relations (des grosses unités constitutives) $)^{71}$, que des « briques » narratives. Le mythe, dès l'origine, en Égypte, n'aurait pas de forme fixe; il serait constitué par des éléments simples que le narrateur ou le ritualiste peut utiliser à sa guise, ce que Miriam Lichtheim appelait «mythological material $»^{72}$. Il importe de l'observer aussi dans les ancrages territoriaux qui sont les siens, dans l'expression complexe des horizons religieux propres aux régions articulées aux données théologiques collectives, sujettes à maintes variations ${ }^{73}$.

\section{MYTHOLOGIES EN CONTACT}

En ce qui concerne la question de la mise en écriture des mythes en Égypte, il faut aussi tenir compte de données propres à l'époque hellénistique. C'est précisément l'époque où, dans la culture grecque, le mythe se trouve subordonné à une enquête de type scientifique et devient un objet d'érudition. Cette démarche - celle de (pseudo-)Apollodore et de sa fameuse «bibliothèque » par exemple - inaugure ce qui sera l'objet de la curiosité des «antiquaires »: la «mythologie $»^{74}$. Or, une démarche analogue existe aussi en Égypte, précisément à l'époque hellénistique; non seulement du côté grec, autour de la bibliothèque d'Alexandrie, mais aussi dans la culture égyptienne contemporaine, d'une tout autre manière. Là également, cette démarche témoigne d'une réflexion systématique sur le mythe débouchant sur sa mise en écriture et révèle, au-delà de la matière mythique, un besoin culturel de recension comme de mise en écriture de cette matière ${ }^{75}$.

71. Claude Lévi-Strauss, Anthropologie structurale, Paris, Plon, 1958, p. 233-234.

72. Miriam Lichtheim, Ancient Egyptian Literature, t. II, Berkeley-Los AngelesLondres, University of California Press, 1976, p. 197.

73. Joachim Friedrich Quack, «Lokalressourcen oder Zentraltheologie ? Zur Relevant und Situierung geographisch struktierter Mythologie im Alten Ägypten », Archiv für Religionsgeschichte, t. 10, 2008, p. 5-29.

74. Jean Starobinski, Le remède dans le mal. Critique et légitimation de l'artifice à l'âge des Lumières, Paris, Gallimard, 1989, spécialement chapitre VI, «Fable et mythologie aux XVII et XVIII siècles ».

75. Jürgen Osing, «Vocabulaires et manuels sacerdotaux à l'époque romaine », Aspects de la culture pharaonique. Quatre leçons au Collège de France, Paris, De Boccard ( Mémoires de l'Académie des Inscriptions et Belles-Lettres », N.S., t. 12), 1992, p. 37-48. Id., « La science sacerdotale », éd. Dominique Valbelle 
En effet, on peut appréhender ainsi cette sorte de "frénésie de l'écriture » qui semble saisir le clergé égyptien de l'époque tardive, qui, tout en s'ingéniant à explorer toute la richesse du système hiéroglyphique pour en inventer des nouvelles combinaisons graphiques (le dit «ptolémaïque »), couvre littéralement les murs des temples de récits qui, auparavant, ne s'y trouvaient pas. Dans le même temps, les clergés semblent suivre un plan directeur, procédant visiblement d'une volonté de recension des croyances et du savoir sacerdotal. On peut aussi admettre que réfléchir sur le(s) mythe(s) dans le terrain égyptien en partant d'une catégorie hellénique (la mythologie) n'est pas complètement incongru. L'exercice est en tout cas bimillénaire, puisque c'est ce que fait Plutarque : dans son traité sur Isis et Osiris, il pratique lui-même le comparatisme ; on oserait dire qu'il pratique déjà, en ses prémices, une «science » des religions, si ce n'est l'analyse structurale des mythes, comme le pensait Claude Lévi-Strauss ${ }^{76}$. Le mythe osirien livré par Plutarque, est, à proprement parler, plutôt un mythe gréco-égyptien, c'est-à-dire très certainement alexandrin. Une vulgate hellénique, un roman lisible pour les Grecs : l'histoire, le cadre sont égyptiens ; la forme, non. Ce qui est donné à lire résulte manifestement du contact entre mythologies. On sait que l'exercice comparatiste fut très largement pratiqué lors de l'Antiquité par les Grecs eux-mêmes, lorsque ceux-ci découvrirent le monde égyptien; il le fut aussi, mais d'une autre façon, par certains Égyptiens, au contact des mythes grecs. Nous sommes ici dans le thème des interprétations mutuelles, où les mythes réagissent entre eux. Il faut constater que cet aspect-là de la question n'a pas été réellement intégré dans le « dossier du mythe » égyptien ; pourtant, il s'y trouve impliqué d'une certaine manière. Chez Hérodote - qui traite non de muthos mais bien de hieros logos - les prêtres égyptiens racontent effectivement des mythes grecs, mais nettement repensés dans le cadre du paysage nilotique. Des mythes grecs qui seraient donc réélaborés par les Égyptiens eux-mêmes,

et Jean Leclant, Le Décret de Memphis. Colloque de la Fondation Singer-Polignac à l'occasion de la célébration du bicentenaire de la découverte de la Pierre de Rosette, Paris, De Boccard, 1999, p. 127-140. Voir aussi Jean Yoyotte, «La science sacerdotale égyptienne à l'époque gréco-romaine ", Revue de l'histoire des religions, t. 159, 1961, p. 133-138.

76. Claude Lévi-Strauss, L’homme nu, Paris, Plon, 1971, p. 18 n.1. 
pour positionner l'Égypte dans l'horizon mythologique $\operatorname{grec}^{77}$. Une réponse égyptienne à la mythologie grecque, qui a été moins questionnée par les spécialistes que la vision grecque de l'Égypte ${ }^{78}$. Simultanément, d'ailleurs, les Grecs reconnaissaient aussi leurs propres mythes en Égypte, même reconstruits par le filtre égyptien. Ce processus d'interprétation s'incarne dans des supports variés, dans des textes, bien sûr, mais également dans des images : il peut, en outre, mettre en jeu un cadre architectural. En voici un exemple, parmi d'autres. Sur une colonne de la cour ramesside du temple de Louqsor est sommairement gravé un personnage masculin, de face, portant un arc. L'inscription le désigne comme étant un «Pâris ». Or, on ne peut manquer de remarquer que ce «Pâris » fait face à une autre image, sculptée à quelques dizaines de centimètres, représentant une reine Nefertari délicatement sculptée, faisant partie de la partie inférieure d'une statue colossale de Ramsès II. Il faut probablement s'imaginer que, dans l'esprit du graveur, ce Pâris aurait retrouvé ici son Hélène, sous forme de reine égyptienne ${ }^{79}$. Sûrement, ce dessin témoigne du fait qu'un visiteur de culture hellénique a pu lire un détail ici d'un temple du Nouvel Empire, selon son bagage mythologique hellénique, qui prenait littéralement vie dans les temples d'Égypte. Au demeurant, il s'agirait du seul témoignage non littéraire attestant la visite en Égypte d'Hélène. Néanmoins, l'association entre la statue de Nefertari et l'eidolon d'Hélène n'est pas certaine, comme l'a souligné Mary Beard ${ }^{80}$. On

77. Cf. Ian S. Moyer, « Herodotus and an Egyptian mirage : the Genealogies of the Theban Priests ", Journal of Hellenic Studies, t. 122, 2002, p. 70-90, et récemment id., Egypt and the Limits of Hellenism, Cambridge, Cambridge University Press, 2011. Cf., dans une autre perspective, Claude Obsomer, «Hérodote et les prêtres de Memphis », Egyptian Religion. The Last Thousand Years, t. II, éd. Anton Schoors, Harco Willems et Willy Clarysse, Louvain, Peeters («Orientalia Lovaniensia Analecta », t. 85), 1998, p. 1423-1442.

78. Alan B. Lloyd, «Herodotus on Egyptians and Libyans », Hérodote et les peuples non grecs, éd. Giuseppe Nenci et Olivier Reverdin, Vandœuvres-Genève, Fondation Hardt («Entretiens sur l'Antiquité classique », t. 35), 1990, p. 215-253.

79. L'intérêt de cette inscription avait été signalé par William Murnane, « Hélène, égyptienne?», Louqsor. Temple du ka royal, Dossiers Histoire et Archéologie, t. 101, 1986, p. 40. Récemment, Colleen Manassa y a consacré une intéressante étude : "A Depiction of Paris in Luxor temple and the "eidolon" of Helen ", Zeitschrift für Ägyptische Sprache und Altertumskunde, t. 136, 2009, p. 141-149.

80. Mary Beard, "The Face of Paris and Cleopatra», The Times Literary Supplement (https://www.the-tls.co.uk/the-face-of-paris-and-cleopatra). Je remercie David Klotz qui m'a signalé cette référence. 
pourrait aussi proposer Aphrodite. Quoi qu'il en soit, cet exemple serait à verser aux dossiers des monuments égyptiens dont les Grecs réécrivent l'histoire, relue par la mythologie classique (le Labyrinthe du Fayoum, le colosse de Memnon à Thèbes, etc.). Cet espace interculturel du mythe, qui relève de l'interpretatio, nous mène, bien entendu, à une autre discussion, qui passe aussi par la prise en compte des images.

Que conclure, à présent, de ce rapide tour d'horizon ? J'ai voulu simplement rappeler comment le « mythe » s'est incontestablement affirmé, tout du long $\mathrm{du} \mathrm{xx}^{\mathrm{e}}$ siècle, comme un objet central d'investigation pour la compréhension de la culture égyptienne. Mais, comme je l'ai déjà évoqué, et pour suivre le constat que faisait Pascal Vernus ${ }^{81}$, il faudrait, bien entendu, s'entendre au départ sur ce qu'est, à l'heure actuelle pour l'histoire des religions, un «mythe». Un questionnement qui pourrait nous amener à nous demander si cette catégorie un peu fourre-tout ne relève pas, à l'instar de bien d'autres concepts, de la science «d'hier ${ }^{82}$. La discussion n'est pas près d'être achevée. Même si l'on postule l'universalité du mythe (comme, autrefois, Roger Caillois ou Mircea Eliade, et bien d'autres) ${ }^{83}$, il n'en demeure pas moins que le mot est grec, ce qui n'est pas sans conséquences, comme le soulignait Jean-Pierre Vernant :

«Par son origine et son histoire, la notion de mythe que nous avons héritée des Grecs appartient à une tradition de pensée qui est propre à l'Occident, et où le mythique se définit par ce qui n'est pas lui, en un double rapport d'opposition au réel d'une part (le mythe est fiction), au rationnel ensuite (le mythe est absurde). C'est dans cette ligne de pensée, dans le cadre de cette tradition, qu'il faut situer, pour

81. P. Vernus, « La notion de mythe dans la civilisation pharaonique », 2001.

82. Je relève, en passant, que nous ne trouvons pas d'entrées «Myth» dans : Mark C. Taylor (éd.), Critical Terms for Religious Studies, Chicago-Londres, University of Chicago Press, 1998. Dans l'index, p. 313, le mot figure, mais sans renvoyer à une quelconque discussion sur sa portée. En revanche, les antiquisants ne savent pas s'en passer, mais s'engagent désormais dans une approche critique du concept, cf. notamment Fritz Graf, « Myth », Religions of the Ancient World, éd. Sarah Iles Johnston, Cambridge (Mass.)-Londres, The Belknap Press of Harvard University Press, 2004, p. 45-58.

83. Au demeurant, Mircea Eliade était très conscient du problème de vocabulaire posé par ce terme, cf. ses Aspects du mythe, Paris, Gallimard, 1963 (passim). 
le comprendre, le développement des études mythiques à l'époque moderne $»^{84}$.

Il y a plus de trente ans Marcel Detienne attirait l'attention sur le fait que la «mythologie », en tant qu'objet grec par essence, avait influé puissamment sur les idées de tous les savants et exégètes occidentaux :

«La mythologie, ça s'écrit. Il n'y a de mythologies, au sens grec, que mythographique, dans l'écriture, par l'écriture, qui en trace les frontières, qui en dessine la figure. Et c'est par illusion que la mythologie est devenue, depuis le $\mathrm{XIX}^{\mathrm{e}}$ siècle, et la parole, et le chant, et la voix des origines $(\ldots) »^{85}$.

Pour Detienne, ce serait même un «profond malentendu» que de présupposer que l'objet mythe puisse être perçu comme mythe «par tout lecteur dans le monde entier». Detienne, sans doute, engage plus à la prudence qu'au relativisme absolu dans l'approche du mythe. Avant lui, les «Mythologiques» de Claude Lévi-Strauss ou la série « Mythe et épopée » de Georges Dumézil ont brillamment montré aux historiens des religions comment l'on pouvait réfléchir sur les formes d'expression des discours mythiques hors du monde classique et renoncer, en même temps, au moins en partie, au formatage hellénique. Avec Lévi-Strauss, le mythe ${ }^{86}$, qui est fondamentalement langage, va conduire du côté de la musique, avec laquelle il noue «de profondes affinités ${ }^{87}$; avec Dumézil, l'affaire s'oriente du côté de l'idéologie. Nous venons de voir comment les égyptologues ont contribué à leur manière à définir l'objet «mythe » dans leur propre champ.

Du côté de l'anthropologie, on s'avise bien désormais du fait que le mythe ne s'exprime pas seulement dans les récits ou par le biais de l'oralité ; intégré dans la mémoire, il passe aussi par divers

84. Jean-Pierre Vernant, Mythe et société en Grèce ancienne, Paris, Maspero, 1974, p. 195.

85. Marcel Detienne, «Repenser la mythologie », La fonction symbolique, éd. Michel Izard et Pierre Smith, Paris, Gallimard, 1979, p. 71-82 (p. 81-82 pour la citation).

86. Pour une approche du mythe chez Lévi-Strauss, voir par exemple : Marcel Hénaff, Claude Lévi-Strauss, Paris, Belfond, 1991, p. 170-200.

87. Claude Lévi-Strauss, Le cru et le cuit. Mythologiques (1), Paris, Plon, 1964, p. 23. 
supports «mnémoniques », comme les appelle Carlo Severi ${ }^{88}$. Cela invite à nous tourner encore vers les images. On s'y est beaucoup intéressé dans une perspective sémiologique ${ }^{89}$; il reste encore à tenter d'en tenir compte dans les théories sur le mythe en Égypte. En effet, si la narration par le texte est souvent introuvable, en revanche les images parlent, à condition que l'on parvienne à les assembler dans un ensemble signifiant. Cette démarche donne des résultats certains : les objets du monde cosmétique, où tout un petit panthéon lié à Hathor ou aux Bès se déploie, livrent les contours de mythes liés à la naissance comme à la renaissance, et à la protection de la vie. On pensera notamment à ce sujet à l'étude magistrale de Jan Quaegebeur sur la barque d'albâtre de Toutankhamon ${ }^{90}$, montrant les bénéfices de l'exploitation maximale des sources iconographiques, ou encore aux nombreux travaux de Jeanne Bulté sur les faïences, partant de l'analyse d'objet, et dévoilant les contours de mythes jamais révélés par les textes ${ }^{91}$.

youri.volokhine@unige.ch

\section{Addendum :}

Sur le mythe en Égypte, voir le livre de Dimitri Meeks, Les Égyptiens et leurs mythes, Paris, Hazan, Chaire du Louvre, 2018, encore inédit lorsque cet article était sous presse.

88. Carlo Severi, Le principe de la chimère. Une anthropologie de la mémoire, Paris, Aesthetica et Musée du Quai Branly, 2007.

89. Cf. par exemple Roland Tefnin, «Approche pour une sémiologie de l'image égyptienne », Chronique d'Égypte, t. 66, 1991, p. 60-88, ou Orly Goldwasser, Prophets, Lovers, and Giraffes: Wor(l)d Classification in Ancient Egypt, Wiesbaden, Harrassowitz (« Göttinger Orientforschungen », t. 38), 2002.

90. Jan Quaegebeur, La naine et le bouquetin, ou l'énigme de la barque en albâtre de Toutankhamon, Louvain, Peeters, 1999.

91. Par exemple, Jeanne Bulté, Talismans égyptiens d'heureuse maternité : «faïence » bleu-vert à pois foncés, Paris, éditions du CNRS, 1991. 